ARTICLE

Received 18 Nov 2015 | Accepted 5 Sep 2016 | Published 12 Oct 2016

DOI: $10.1038 /$ ncomms13125

OPEN

\title{
Specification of haematopoietic stem cell fate via modulation of mitochondrial activity
}

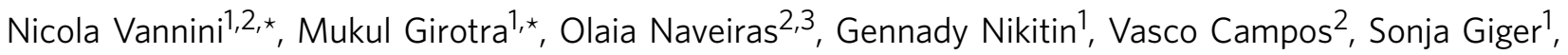
Aline Roch ${ }^{1}$, Johan Auwerx ${ }^{4} \&$ Matthias P. Lutolf ${ }^{1,5}$

Haematopoietic stem cells (HSCs) differ from their committed progeny by relying primarily on anaerobic glycolysis rather than mitochondrial oxidative phosphorylation for energy production. However, whether this change in the metabolic program is the cause or the consequence of the unique function of HSCs remains unknown. Here we show that enforced modulation of energy metabolism impacts HSC self-renewal. Lowering the mitochondrial activity of HSCs by chemically uncoupling the electron transport chain drives self-renewal under culture conditions that normally induce rapid differentiation. We demonstrate that this metabolic specification of HSC fate occurs through the reversible decrease of mitochondrial mass by autophagy. Our data thus reveal a causal relationship between mitochondrial metabolism and fate choice of HSCs and also provide a valuable tool to expand HSCs outside of their native bone marrow niches.

\footnotetext{
${ }^{1}$ Laboratory of Stem Cell Bioengineering, Institute of Bioengineering, School of Life Sciences and School of Engineering, Ecole Polytechnique Fédérale de Lausanne (EPFL), CH-1015 Lausanne, Switzerland. ${ }^{2}$ Laboratory of Regenerative Hematopoiesis (GR-NAVEIRAS), Institute of Bioengineering, School of Life Sciences, Ecole Polytechnique Fédérale de Lausanne (EPFL), CH-1015 Lausanne, Switzerland. ${ }^{3}$ Departments of Medicine and Oncology, Centre Hospitalier Universitaire Vaudois (CHUV), CH-1011 Lausanne, Switzerland. ${ }^{4}$ Laboratory of Integrative and Systems Physiology, Institute of Bioengineering, School of Life Sciences, Ecole Polytechnique Fédérale de Lausanne (EPFL), CH-1015 Lausanne, Switzerland. ${ }^{5}$ Institute of Chemical Sciences and Engineering, School of Basic Science, Ecole Polytechnique Fédérale de Lausanne (EPFL), CH-1015 Lausanne, Switzerland. * These authors contributed equally to this work. Correspondence and requests for materials should be addressed to M.P.L. (email: matthias.lutolf@epfl.ch).
} 
T he maintenance of the blood system is ensured by a pool of HSCs residing in hypoxic niches in the bone marrow $(\mathrm{BM})^{1}$. These unique cells are capable of lifelong self-renewal and commitment to multipotent progenitors (MPP). For many decades, HSCs have been successfully used for treating haematological and immune diseases. However, their limited number, especially when isolated from umbilical cord, prevents a more reliable and broader application of HSC-based therapies $^{2-4}$. Despite recent notable success stories ${ }^{5,6}$, many attempts to propagate HSCs in vitro have failed, primarily because self-renewal and in vivo regenerative capacity is rapidly lost in culture.

Recent studies have shown that the change in cell identity and function during early HSC commitment involves a profound alteration in the metabolic program of the cells. Long-term HSCs (LT-HSCs) are mostly quiescent and tend to produce energy preferentially by anaerobic glycolysis ${ }^{1,7,8}$, which has been linked to their residence in low oxygen niches ${ }^{9,10}$. In contrast, the stem and progenitor cell types that produce blood and have a reduced self-renewal ability (that is, short-term HSCs and rapidly proliferating MPPs) generate ATP primarily in the mitochondria by oxidative phosphorylation (OXPHOS) ${ }^{7,11}$. The distinct metabolic program of LT-HSCs appears to play a critical role in maintaining their long-term in vivo function, presumably because the reduced mitochondrial respiration protects the cells from cellular damage inflicted by reactive oxygen species (ROS) in active mitochondria ${ }^{12-16}$.

The metabolic switch that occurs during the earliest step of adult haematopoiesis suggests a direct role of mitochondria in regulating HSC fate. This hypothesis is supported by work demonstrating that a metabolic transducer, the tumour suppressor and glucose sensor Lkb1 is crucial for HSC maintenance ${ }^{16-19}$. Moreover, autophagy, through which cells can modulate mitochondrial numbers, has been shown to improve HSC maintenance ${ }^{20}$. However, whether the metabolic state of HSCs is more than an adaptation to an extreme microenvironment in the $\mathrm{BM}$, and perhaps linked to the ability to execute a particular cell fate choice, is currently not known.

Here we used the mitochondrial activity as a surrogate for the metabolic state of HSCs. Using in vivo multi-lineage blood reconstitution assays, we show that long-term self-renewal activity is restricted to phenotypic HSC subpopulations having lower mitochondrial activity. By comparing mitochondrial activity distributions of HSCs separated by their cell cycle phase, we find that during homeostasis as well as under acute stress, quiescent and cycling HSCs have relatively similar mitochondrial activity profiles. This shows that the distinct metabolic programs of HSCs are rather indicative of fate choice (that is, self-renewal versus commitment) and not per se a hallmark of the quiescent (versus activated) state. Indeed, in vitro, in heterogeneous HSC expansion cultures, divisional tracking experiments show that actively self-renewing HSCs retain a low mitochondrial activity, in marked contrast to differentiating cells that have activated mitochondria. Finally, under differentiation culture conditions, the chemical uncoupling of the electron transport chain forces HSC to self-renew, a process that is accompanied by a reversible decrease of mitochondrial mass by autophagy. Collectively, our experiments reveal an intriguing relationship between HSC metabolism and fate decision-making.

\section{Results}

HSCs show low mitochondrial activity and mass. To establish a reliable marker for tracking the metabolic state of HSCs undergoing different fate choices, we first employed flow cytometry and confocal microscopy to analyse the mitochondrial activity and mass of phenotypically defined HSC and MPP populations that were isolated by the most commonly used combinations of surface markers ${ }^{21,22}$ (Supplementary Fig. 1). We used tetramethylrhodamine methyl ester (TMRM), a cell-permeable dye that is readily sequestered by active mitochondria and reports mitochondrial polarization that correlates with the level of OXPHOS in a cell ${ }^{23,24}$. Each population displayed a distinct level of TMRM intensity, giving a stepwise increase from the most primitive to the most committed population as shown by flow cytometry (Supplementary Fig. 1a,b) and microscopy-based read-outs (Supplementary Fig. 1c,d). The mitochondrial activity was lowest in the LT-HSC population (Supplementary Fig. 1b), which was suspected based on recent studies reporting a preferential use of glycolysis in LT-HSCs ${ }^{7,11,25}$, and became barely detectable by confocal microscopy (Supplementary Fig. 1c,d).

We then asked whether HSCs and MPPs also show differences in their mitochondrial mass. To this end, we employed an R26-mito-EGFP mouse line $e^{26}$ ubiquitously expressing enhanced green fluorescent protein (EGFP) in the mitochondria of each cell. An analysis of single cells isolated from these mice by spinning disk microscopy revealed the lowest level of mitochondrial mass in the LT-HSC compartment (Supplementary Fig. 1e,f). This is in accordance with previous work showing that blocking HSC differentiation by TSC1-mediated mTOR pathway inhibition is accompanied by a decrease in mitochondrial mass ${ }^{15}$. Additionally, FOXO3, a transcription factor critical for the LT-HSC self-renewall ${ }^{27}$, promotes mitochondrial mass reduction ${ }^{28}$. Thus, these results confirm that under homeostatic conditions, freshly isolated LT-HSCs are marked by both low mitochondrial activity and mass.

Low mitochondrial activity marks LT-HSCs. Using in vivo multi-lineage blood reconstitution assays, we next used phenotypically defined LKS (a population that contains all multipotent stem and progenitor cells in the BM, thus also the putative HSCs), ST- or LT-HSCs to test to which extent mitochondrial activity levels could report stem cell function (Fig. 1). First, we focused on LKS and utilized FACS to isolate two cell fractions within the LKS compartment characterized by low (LKS:TMRM ${ }^{\text {low }}$ ) and high (LKS:TMRM ${ }^{\text {high }}$ ) TMRM intensity levels. Then, we transplanted these two metabolically different cell populations into lethally irradiated mice by using a double congenic allelic system (Fig. 1a). Long-term multi-lineage blood reconstitution analysis showed that within the LKS population, only cells with low TMRM intensity (that is, LKS:TMRM ${ }^{\text {low }}$ ) permitted long-term multi-lineage reconstitution (Fig. 1b,c). Therefore, employing a metabolic read-out along with the existing surface marker repertoire allows purification of cells with long-term reconstitution capacity from a poorly defined population (LKS) consisting mainly of MPPs.

Then, using the same sorting strategy on ST-HSCs, setting the TMRM gates as shown in Supplementary Fig. 2, we compared the ability of ST-HSC:TMRM ${ }^{\text {low }}$ and ST-HSC:TMRM ${ }^{\text {high }}$ to reconstitute the blood system (Fig. 1d,e). Strikingly, within the ST-HSC population, the capacity to reconstitute the blood system was almost exclusively restricted to the TMRM ${ }^{\text {low }}$ fraction (Fig. 1e). Moreover, sorted LT-HSCs (Fig. 1d) could similarly be separated into two functionally very distinct populations. Only the population with low mitochondrial activity (LT-HSC:TMRM $^{\text {low }}$, corresponding to $\sim 55 \%$ of the population) was capable of long-term multi-lineage reconstitution, whereas LT-HSC:TMRM ${ }^{\text {high }}$ cells completely failed to do so (Fig. 1f). Importantly, propidium iodide staining did not show any measurable difference in viability between the four populations 
a

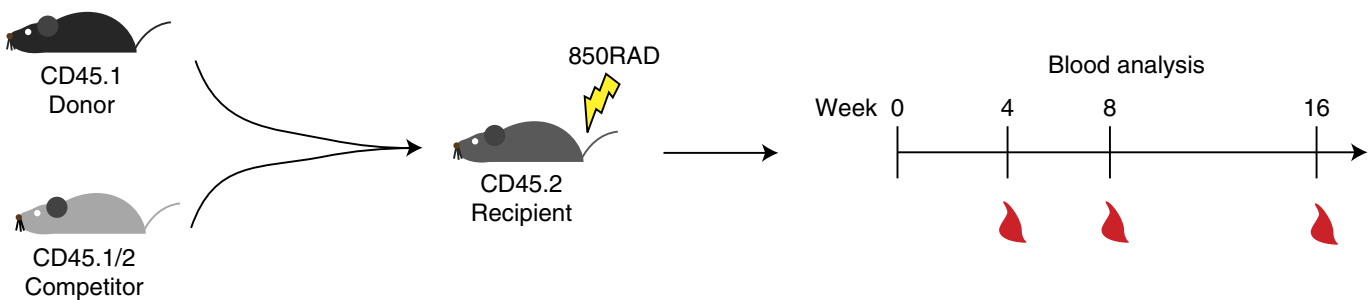

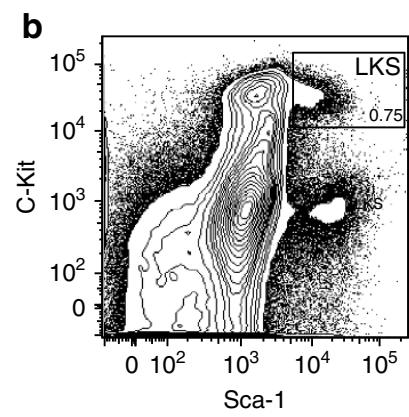

d
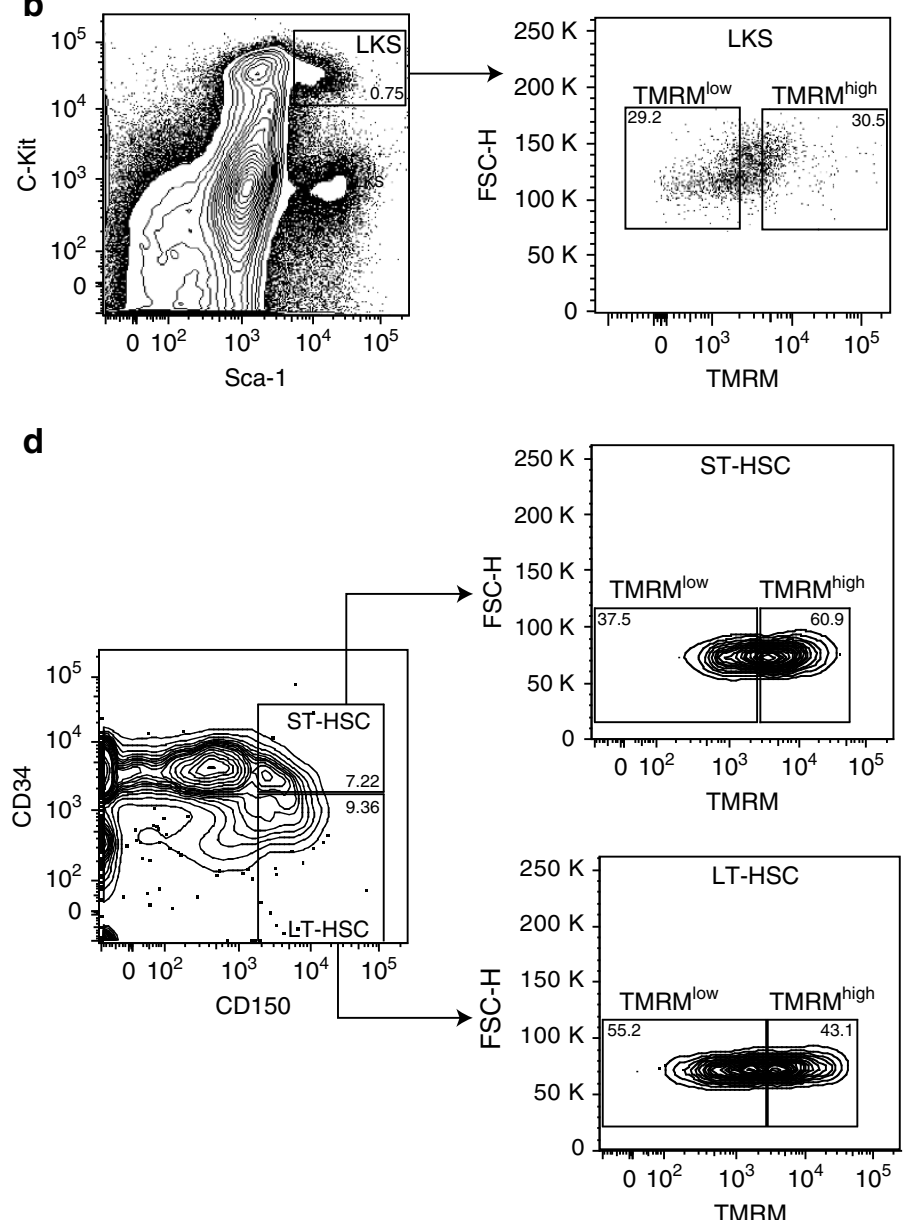

C
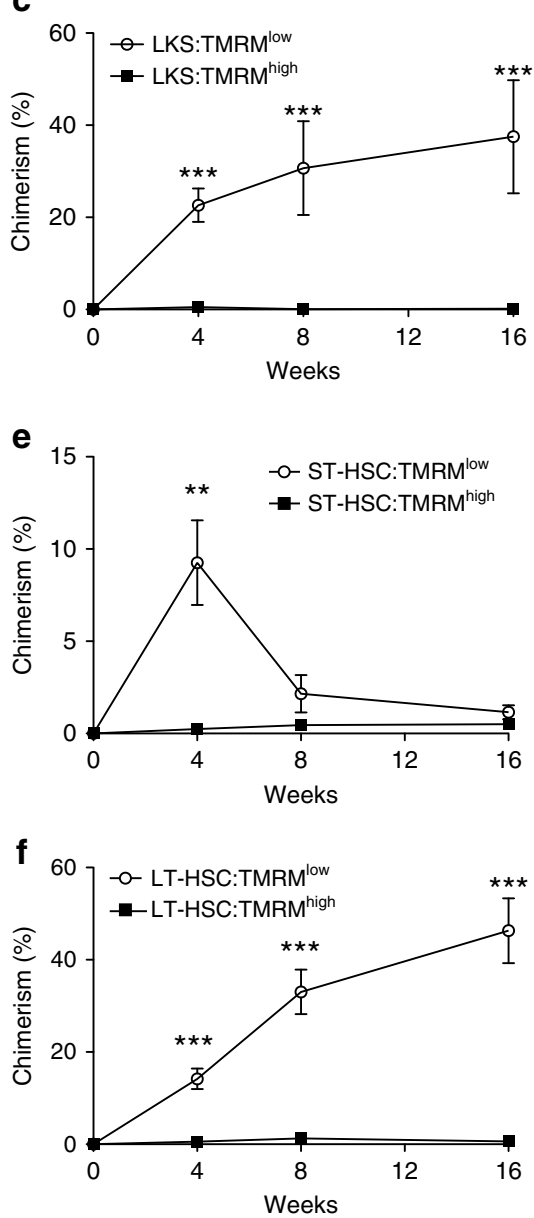

Figure 1 | Multi-lineage reconstitution capacity is restricted to the low mitochondrial activity cell fractions. (a) Competitive transplantation strategy used to assess multi-lineage blood reconstitution levels from peripheral blood after 4, 8 and 16 weeks. (b,c) Within LKS, which contain all multipotent stem and progenitor cells in the BM, long-term multi-lineage HSC function is restricted to TMRM ${ }^{\text {low }}$ cells (LKS:TMRM ${ }^{\text {low }}$ ) ( $n=8$ for each condition; error bar: s.e.m.; $t$-student, $\left.{ }^{\star \star \star} P<0.001\right)$. (d,e) In the phenotypically defined ST-HSC compartment, stemness is restricted to TMRM ${ }^{\text {low }}$ cells (ST-HSC:TMRM ${ }^{\text {low })}$ ( $n=9$ for each condition; error bar: s.e.m.; $t$-student, $\left.{ }^{\star \star} P<0.01\right)$. (d,f) In the phenotypically defined LT-HSC compartment, stemness is restricted to TMRM $^{\text {low }}$ cells (LT-HSC:TMRM ${ }^{\text {low }}$ ) $\left(n=9\right.$ for each condition; error bar: s.e.m.; $t$-student, $\left.{ }^{\star \star \star} P<0.001\right)$.

(Supplementary Fig. 3), and BM isolated based on differential TMRM levels did not show significant differences in engraftment capability (Supplementary Fig. 4), ruling out that the lack of engraftment was a result of differential cell death. Moreover, we compared the homing capacity of transplanted cells and did not find any difference between HSCs isolated based on differential mitochondrial activity levels (Supplementary Fig. 5).

Our in vivo data thus reveal a striking functional heterogeneity in phenotypically defined HSCs. The observation that LT-HSCs with activated mitochondria (that is, LT-HSC:TMRM ${ }^{\text {high }}$ ) do not show a significant blood reconstitution suggests that these cells may not be hierarchically related to 'true' LT-HSCs. They may instead represent HSCs that give rise to long-term lineage-restricted progenitor cells, as shown by in vivo singlecell multi-lineage reconstitution assays performed on the same immunophenotypes ${ }^{29}$. To test whether TMRM ${ }^{\text {low }}$ versus TMRM ${ }^{\text {high }}$ cells within the LT-HSC compartment contain different levels of such lineage-biased progenitor cells, we used CD41, a marker for megakaryocytes, to further separate the two phenotypes. Previous studies have shown that CD41 + cells in the HSC compartment have a strong myeloid bias ${ }^{30}$ and only short- or intermediate-term repopulation activity ${ }^{29}$. In our hands, LT-HSC:TMRM ${ }^{\text {low }}$ cells comprise $\sim 70 \%$ of cells that are negative for CD41, in marked contrast to LT-HSC:TMRM ${ }^{\text {high }}$ cells, of which $\sim 50 \%$ are CD41 positive (Supplementary Fig. 6). Therefore, the observed functional heterogeneity may at least be 
partially explained by the presence of CD41 + megakaryocyte progenitors that might have different mitochondrial activity levels compared with functional LT-HSCs.

Low mitochondrial activity marks rare self-renewing HSCs. After establishing low mitochondrial activity as a functional discriminator of stemness, we tested whether this read-out could be used to distinguish self-renewing from differentiating HSCs in heterogeneous bulk cultures. To test whether TMRM-based read-outs could be used to separate self-renewal from differentiation divisions, 200 LT-HSCs from CD45.1 donor mice were isolated based on surface marker expression and expanded in vitro in serum-free medium containing a cocktail of the self-renewing factors angiopoietin-like protein, insulin-like growth factor binding protein 2, stem cell factor, fibroblast growth factor 1 and thrombopoietin ${ }^{31}$ (Supplementary Fig. 7). After 5 days in culture, the cells were resorted by FACS based on TMRM $^{\text {low }}$ and TMRM ${ }^{\text {high }}$ phenotypes, and transplanted into lethally irradiated CD45.2 recipient mice (Supplementary Fig. 7a). Of note, we found that the TMRM intensities of cultured cells are substantially different from those of freshly isolated cells (Fig. 1b), likely reflecting an influence of cell culture. Nonetheless, consistent with our previous results using low TMRM fluorescence to directly isolate functional HSCs in vivo, a low TMRM signal was also predictive of the long-term blood reconstitution capacity of cultured HSCs (Supplementary Fig. 7b). Analysis of peripheral blood chimerism 4 months after grafting the cultured HSCs showed that TMRM ${ }^{\text {low }}$ cells induce significantly higher long-term multi-lineage blood reconstitution levels compared with cells that had a high TMRM signal. This suggested that self-renewing HSCs in culture can be detected based on the same metabolic hallmark as freshly isolated HSCs from the BM.

However, since it is known that a small fraction of cultured HSCs can maintain their stem cell potential by remaining quiescent even after prolonged time in culture (for example, refs 32,33 ), the reconstitution from TMRM ${ }^{\text {low }}$ cells seen in Supplementary Fig. 7 might have come from non-dividing HSCs. To exclude this possibility and unequivocally identify selfrenewing cells in these bulk cultures, we created a cell-labelling strategy that allowed us to track the precise number of divisions a 'mother' HSC had undergone in culture (Fig. 2a,b). Freshly isolated LT-HSCs were uniformly labelled with carboxy-fluoresceinsuccinimidyl ester (CFSE), a live cell-permeable dye that allows cell division tracking ${ }^{34}$. With this tool, we FACS-sorted cultured HSC daughter cells that underwent precisely one division after 2 days and then further separated them into TMRM $^{\text {low }}$ and TMRM ${ }^{\text {high }}$ subpopulations (Fig. 2b, lower panels). Hundred cells of each population were transplanted into lethally irradiated mice and long-term multi-lineage reconstitution was analysed up to 16 weeks later. In line with our previous findings, long-term multi-lineage reconstitution was once again restricted to the TMRM ${ }^{\text {low }}$ subpopulation (Fig. 2c). Furthermore, secondary transplantation showed long-term engraftment of the TMRM ${ }^{\text {low }}$ fraction (Fig. 2d). These data establish low mitochondrial activity as a read-out for self-renewal divisions of HSCs in vitro. Moreover, our experiments reveal that low mitochondrial activity is a hallmark not only of quiescent LT-HSC in vivo, as could be hypothesized based on their metabolic state of preferentially undergoing anaerobic glycolysis ${ }^{9,10}$, but also of cycling stem cells that have undergone a self-renewing cell division in vitro. Notably, a cell cycle phase analysis of HSCs in vivo showed that quiescent and cycling LT-HSCs have similar levels of mitochondrial activity, both under homeostatic conditions (Fig. 3a,b) and when stimulated by interferon-alpha
(IFN- $\alpha$ ) to exit dormancy ${ }^{35}$ (Fig. 3c,d) and maintaining stem cell potential (Supplementary Fig. 8). In the latter experiments, analysis of mitochondrial activity combined with cell cycle phase analysis was performed by MitoTracker Deep Red staining that persists in the cells after fixation ${ }^{10,36}$ and gave consistent readouts on the metabolic profiles of the different haematopoietic compartments (Supplementary Fig. 9).

HSC fate specification by electron transport chain uncoupling. With a read-out identified to discriminate HSC self-renewal from differentiation, we then tested whether HSC fate could be manipulated by modulation of mitochondrial activity in vitro (Fig. 4). Specifically, we chose culture conditions that would rapidly push HSCs to differentiate into highly proliferative MPPs and asked whether blocking the establishment of a mitochondrial membrane potential would result in the maintenance of stemness (Fig. 4a). For that purpose, we used carbonyl cyanide- $p$ trifluoromethoxyphenylhydrazone (FCCP), which permeabilizes the inner mitochondrial membrane and disrupts its potential (Supplementary Fig. 10), to uncouple electron transport from ATP generation.

Long-term blood reconstitution assays show that LT-HSC: TMRM $^{\text {low }}$ cultured for 5 days under differentiation-inducing conditions (SCF, Flt3, IL-3 and IL-6) and in the presence of FCCP exhibited substantially higher levels of long-term multi-lineage blood reconstitution in lethally irradiated recipient mice, compared with cells cultured in the absence of FCCP (Fig. 4b). By labelling HSCs with CFSE and tracking their divisional history, we could rule out that this effect was due to an induction of quiescence upon FCCP administration, since all cells in culture had divided multiple times (Supplementary Fig. 11). Therefore, by disrupting the inner mitochondrial membrane potential, HSCs that would normally rapidly differentiate can be converted to a state of self-renewing division. These data demonstrate that HSC self-renewal divisions can be executed independently of the establishment of a mitochondrial potential. Interestingly, rapidly dividing, self-renewing embryonic stem cells have mitochondria with a low mitochondrial membrane potential indicative of OXPHOS-independent metabolism ${ }^{37}$. Furthermore, during reprogramming of fibroblasts to the induced pluripotent stem cell state, a metabolic switch from OXPHOS to glycolysis is required ${ }^{24}$. Of note, LT-HSC:TMRM ${ }^{\text {high }}$ treated with FCCP failed to revert to functional stem cells (Supplementary Fig. 12), enforcing the hypothesis that LT-HSC:TMRM ${ }^{\text {high }}$ cells may be composed of more committed haematopoietic cells. Indeed, LT-HSC:TMRM ${ }^{\text {low }}$ are exclusively composed by CD48 - cells, while the TMRM ${ }^{\text {high }}$ fraction contains both CD48 - and CD48 + cells (Supplementary Fig. 13), as well as CD41 + cells (Supplementary Fig. 6).

Chemical uncoupling increases autophagy in HSCs. FCCP is known to promote mitophagy ${ }^{38}$ and, in turn, autophagy has been demonstrated to be critical for LT-HSC self-renewal ${ }^{27,28,39}$. Accordingly, the inhibition of autophagy gives rise to an increase in the mitochondrial mass in LT-HSCs through an accumulation of mitochondria damaged by $\operatorname{ROS}^{20}$. Therefore, we postulated that the observed metabolic specification of HSC fate (Fig. 4b) could occur through a reversible decrease of mitochondrial mass via autophagy. To test this, we used spinning disk confocal imaging at single cell level to analyse whether the treatment of HSCs with FCCP under differentiation-inducing conditions is accompanied by a decrease of mitochondrial mass (TOMM20) and/or elevation of autophagy (LC3B) (Fig. 4c). The rate of autophagosomal turnover was assessed by blocking their lysosomal degradation by Pepstatin A for $4 \mathrm{~h}$ prior to fixation. This analysis showed that chemical uncoupling by 

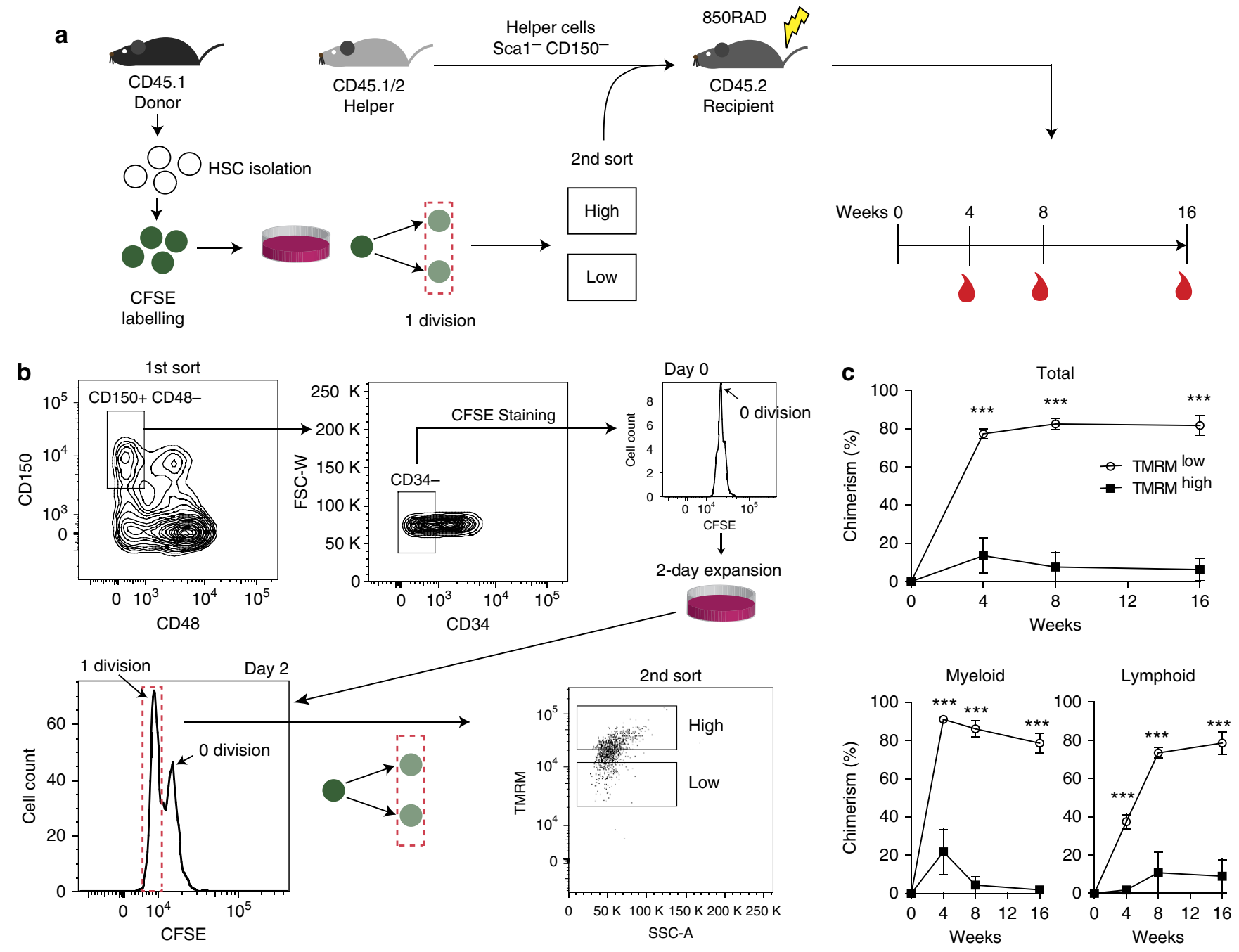

d
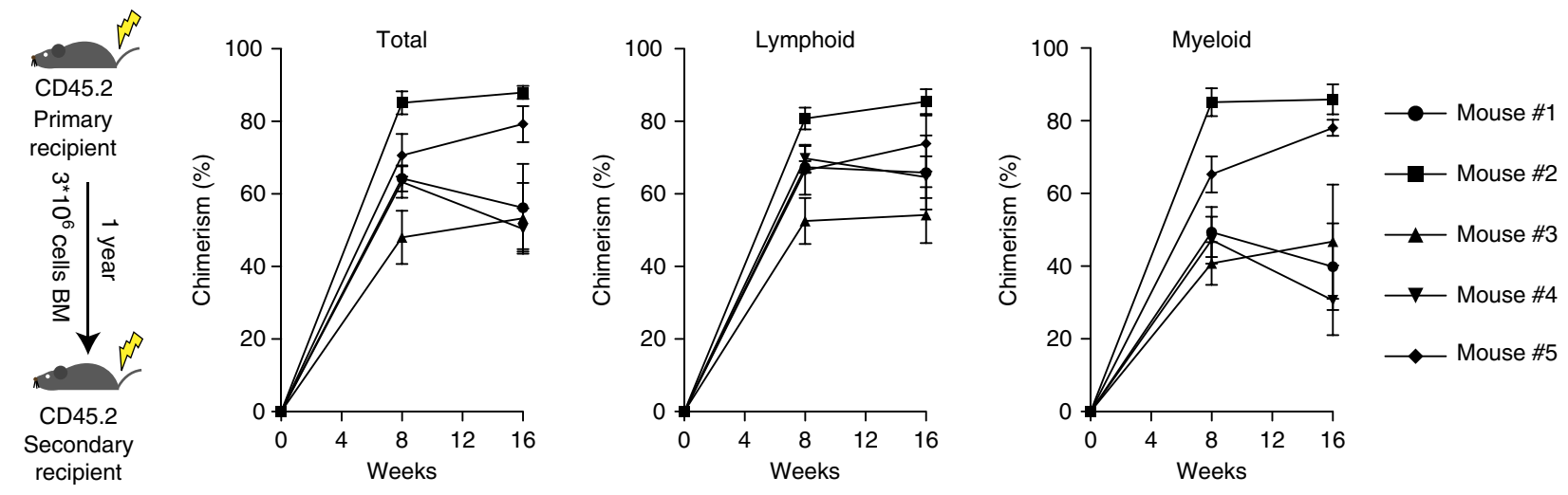

Figure 2 | A low mitochondrial activity marks self-renewing HSCs. (a) Experimental paradigm to assess mitochondrial activity as a discriminator of self-renewing from differentiating LT-HSCs in culture. Freshly isolated LT-HSCs were labelled with CFSE. At the end of the culture, cells that divided one time were further sorted into TMRM ${ }^{\text {low }}$ and TMRM high phenotypes and transplanted into lethally irradiated recipient mice together with helper cells. Blood reconstitution was assessed at 4, 8 and 16 weeks. (b) CFSE-labelled LT-HSCs were cultured under expansion conditions for 2 days and progeny that underwent one division were sorted into TMRM ${ }^{\text {low }}$ and TMRM ${ }^{\text {high }}$ phenotypes, and 100 cells of each population (along with 2 million helper cells) were transplanted into lethally irradiated recipients. (c) The TMRM ${ }^{\text {low }}$ fraction of the first generation of daughter cells (that is, dividing one time) exhibited strikingly higher long-term multi-lineage blood reconstitution efficiency compared with TMRM high cells, providing evidence for self-renewing versus differentiating HSC divisions in culture ( $n=10$ for each condition). Assessment of blood chimerism is shown for total blood (top panel) as well as the lymphoid and myeloid lineages (bottom panels; error bar: s.e.m.; $t$-student, ${ }^{\star \star \star} P<0.001$ ). (d) BM derived from each of the TMRM ${ }^{\text {low }}$ primary recipients (from c) was injected into four secondary recipient mice after 1 year of the primary transplant. Blood chimerism (average of four secondary recipients corresponding to each primary recipient) show long-term multi-lineage reconstitution in secondary transplants (error bar: s.e.m.). 

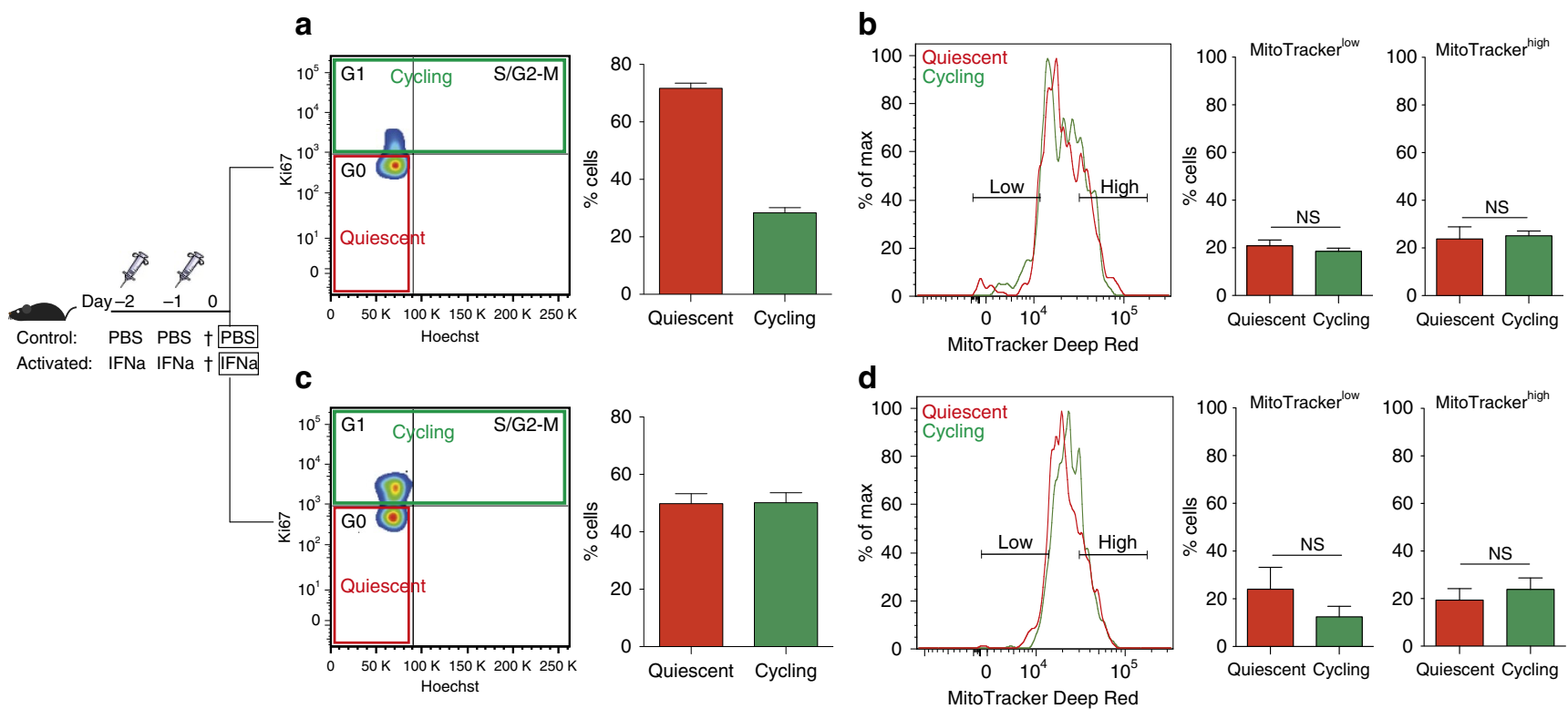

Figure 3 | Quiescent and cycling HSC populations in the native niche have comparable mitochondrial activity levels. (a) Cell cycle analysis using Ki67 and Hoechst staining on freshly isolated HSCs (LKS CD150 + CD48 - CD34 - ) indicate that more than 70\% of the cells are in a quiescent state (G, red), with the remaining cells cycling $\left(G_{1}+S / G_{2}-M\right.$, green) $(n=3)$. (b) Flow cytometry analysis of quiescent and cycling HSCs based on mitochondrial activity labelled with MitoTracker Deep Red. Both, quiescent and cycling HSCs show overlapping mitochondrial activity profiles. The proportion of low and high mitochondrial activity cells within the quiescent and cycling HSC populations is similar (MitoTrackerlow: $P=0.44$, MitoTracker high: $P=0.81 ; n=3$; error bar: s.e.m., $t$-student). (c) IFN- $\alpha$ stimulation results in in vivo activation of HSCs as demonstrated by Hoechst/Ki67 staining ( $n=3$ ). (d) Flow cytometry analysis shows overlapping MitoTracker profiles of quiescent and cycling HSCs in IFN- $\alpha$ condition. Similarly, the proportion of MitoTracker ${ }^{\text {low }}$ and MitoTracker high cells in quiescent and cycling HSCs remains comparable (MitoTrackerlow: $P=0.32$, MitoTracker high: $P=0.54 ; n=3$; error bar: s.e.m., $t$-student), suggesting that mitochondrial activity is independent of HSC cell cycle state even under acute stress conditions.

FCCP drastically decreased the mitochondrial area as measured by TOMM20 in LT-HSC (Fig. 4d). This phenomenon is reversed in the presence of protease inhibitor Pepstatin A, which causes the accumulation of autophagosomes before mitochondrial autophagy can occur. Thus, while autophagosomal area as measured by LC3B immunofluorescence seems similar in FCCP-treated versus control HSCs (Fig. 4e), further treatment with protease inhibitor Pepstatin A shows a drastic increase in LC3B autophagosome accumulation in FCCP-treated cells, but not in control cells. This effect is especially clear, when the autophagosomal area is normalized to the mitochondrial area. The ratio between the autophagosomal and mitochondrial area is higher in FCCP-treated cells compared to control cells, and this effect is further emphasized by the inhibition of lysosomal degradation of the autophagosomes by Pepstatin A (Fig. 4f). Taken together, these results point to the reduction in mitochondrial mass in LT-HSCs upon FCCP treatment as a result of both increased autophagosome formation and accelerated lysosomal processing of the autophagosomes. Consistently, gene expression analysis revealed an increase in expression levels of the E3 ubiquitin ligase PARKIN (encoded by PARK2), involved in the ubiquitination of depolarized mitochondria ${ }^{40}$, and of the autophagic receptor Sqstm $1^{41}$ (Fig. 4g). Therefore, the chemical uncoupling of the electron transport chain increases the level of autophagy in LT-HSCs, and the mitochondria serve as a primary target for the removal by autophagosomes.

\section{Discussion}

Collectively, our data show that in HSCs, mitochondrial metabolism and function are intricately linked. Specifically, we demonstrate that the distinct metabolic program of HSCs is more than an adaptation to a specific microenvironment in the $\mathrm{BM}$ niche ${ }^{9,10}$, but seems sufficient to drive the decision of stem cells to undergo self-renewal or commitment. It should be noted, however, that the mitochondrial activity is a transient cell state that may strongly depend on endogenous conditions such as the microenvironment. Cells may not be readily interconvertible between high and low mitochondrial activity levels (Supplementary Fig. 12).

Using a gene-knockout approach, previous work has shown that loss of function of PTPMT1 phosphatase within the inner mitochondrial membrane is sufficient to block differentiation and promote self-renewal in vivo, apparently through the accumulation of phosphatidylinositol phosphate substrates that enhance endogenous UCP2 activity and lower mitochondrial aerobic metabolism ${ }^{11}$. However, loss of PTPMT1 failed to drive preferential HSC self-renewing divisions in vitro, presumably because of the oxidative stress imposed by classic culture conditions. Loss of PTPMT1 is also an irreversible treatment that results in the inability of stem cells to differentiate in vivo, causing abnormal expansion of the HSC compartment and ultimately a failure of haematopoiesis. Here we show that chemical uncoupling of the electron transport chain is sufficient to reversibly metabolically modulate HSCs via induction of autophagy, maintaining their capacity for proper differentiation and functional blood reconstitution.

We think that our approach could be useful to expand HSCs for clinical applications. Moreover, the availability of a functional marker for HSC self-renewal in the form of low mitochondrial potential as measured by TMRM, extensively validated here for both in vitro and in vivo applications, should facilitate investigations of molecular mechanisms of HSC fate decision-making.

\section{Methods}

Mice. C57Bl/6J and C57Bl/6J Ly5.1 were purchased from Charles River Laboratories International and maintained at the Center for Studying Living System (CAV) at EPFL in micro-isolator cages. Mice were provided continuously with sterile food, water and bedding. All in vivo procedures were carried out in accordance with the Swiss law and EPFL policies. 

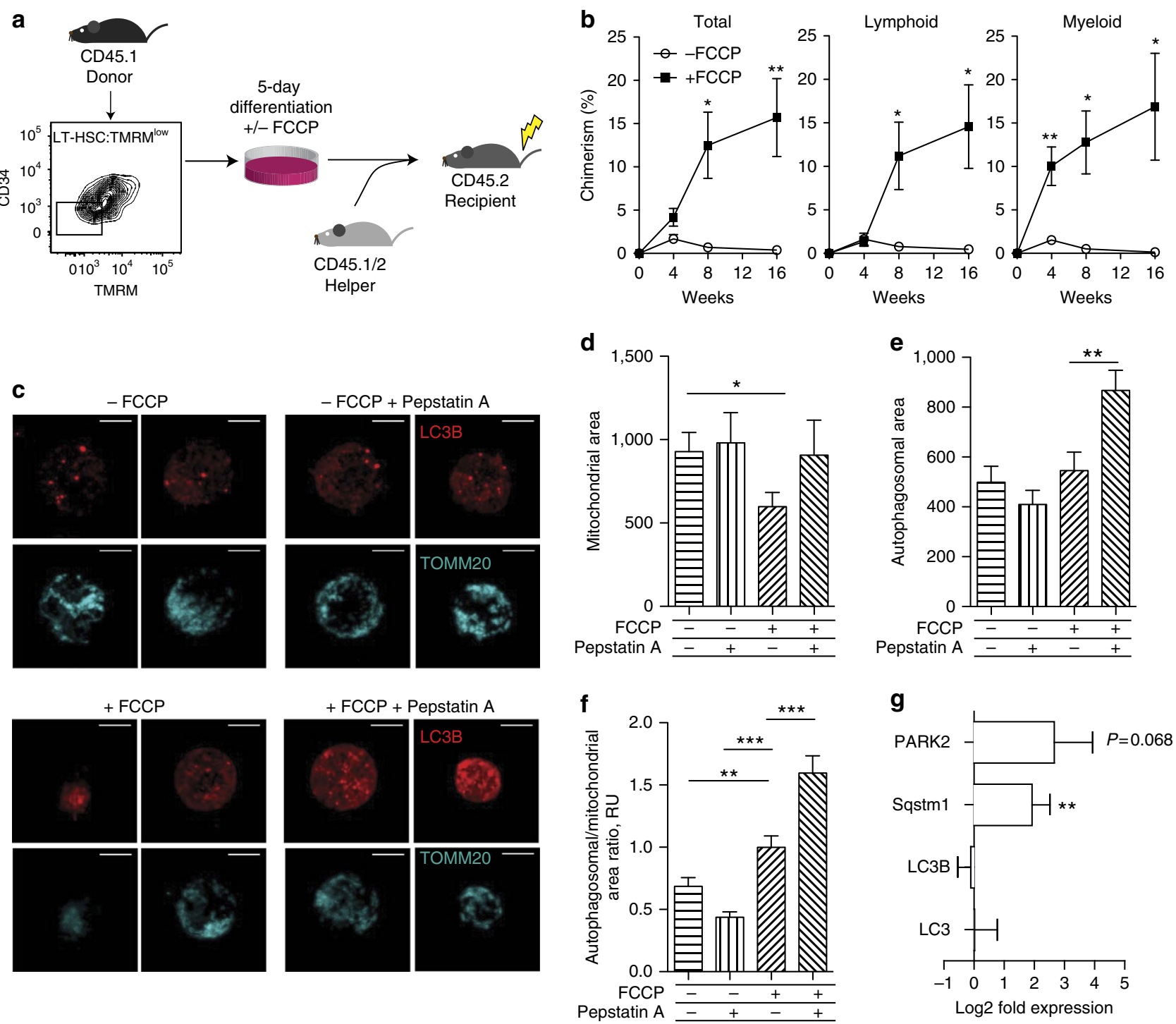

Figure 4 | Modulation of mitochondrial metabolism alters HSC fate through autophagy. (a) LT-HSC:TMRM ${ }^{\text {low }}$ (LKS CD150 + CD34 - TMRM ${ }^{\text {low }}$ ) cultured for 5 days under differentiation conditions in the presence or absence of FCCP were transplanted in lethally irradiated recipient mice together with $2{ }^{\star} 10^{6}$ helper cells. (b) Cells cultured in the presence of FCCP show high levels of multi-lineage reconstitution, in contrast to controls lacking FCCP that result in rapid differentiation (error bar: s.e.m.; $t$-student, ${ }^{\star \star} P<0.01$ and $\left.{ }^{\star} P<0.05\right)$. (c) Mitochondrial mass (TOMM20, cyan) and autophagosomes (LC3B, red) in different haematopoietic stem/progenitor cell populations. Images represent maximum intensity projections of the corresponding Z-stacks $(0.28 \mu \mathrm{m}$ step) Scale bar: $5 \mu \mathrm{m}$. (d-f) Quantification of the mito-EGFP (d), LC3B (e) and mito-EGFP/LC3B ratio intensity-thresholded areas (f). (g) Gene expression analysis on key autophagosomal and mitophagic genes was performed on LT-HSCs treated over 5 days with FCCP (error bar: s.e.m.; $t$-student, ${ }^{\star \star \star} P<0.001,{ }^{\star \star} P<0.01$ and $\left.{ }^{\star} P<0.05\right)$.

Antibodies. The following antibodies were used: rat mAbs against $1 / 200 \mathrm{cKit}$ (2B8) (Biolegend Cat. no. 105814), 1/100 Sca1 (D7) (Biolegend Cat. no. 108112), 1/100 CD150 (TC-15-12F12.2), 1/25 CD34 (RAM34), 1/100 CD48 (HM48-1) (Biolegend Cat. no. 103418), 1/200 CD45.2 (104) (Biolegend Cat. no. 109820), 1/200 CD45.1 (A20) (Biolegend Cat. no. 110706), 1/1,000 Gr1 (RB6-8C5) (Biolegend Cat. no. 108412), 1/750 F4/80 (BM8), 1/500 CD19 (6D5) (Biolegend Cat. no. 115507), 1/200 CD3 (17A2) (Biolegend Cat. no. 100206), 1/500 CD16/CD32 (2.4G2) (BD Cat. no. 553141). The antibodies were purchased from Biolegend, eBiosciences and BD (Becton, Dickinson and Company). A mixture of biotinylated mAbs against CD3, CD11b, CD45R/B220, Ly-6G, Ly-6C and TER-119 was used as lineage cocktail (BD).

Flow cytometry and fluorescence activated cell sorting. Flow cytometry analysis of haematopoietic stem and progenitor cells was performed on freshly isolated BM. BM was extracted from crushed femora and tibia. Cell suspensions were filtered through a $70 \mu \mathrm{m}$ cell strainer and erythroid cells were eliminated by incubation with red blood cells lysis buffer (eBioscences). Lineage-positive cells were removed with a magnetic lineage depletion kit (Miltenyi Biotech). Cell suspensions were stained with a panel of specific antibodies for stem and progenitor cells and analysed or FACS-sorted on a BD LSRII and BD FACS Aria II, respectively.

Analysis of mitochondrial activity. Freshly isolated BM cells were incubated at $37^{\circ} \mathrm{C}$ for $1 \mathrm{~h}$ with $200 \mathrm{nM}$ TMRM (Invitrogen) and then stained with specific antibodies for the different haematopoietic stem/progenitor cell compartments. Labelled cells were FACS-sorted or analysed by flow cytometry. For confocal imaging, LT-HSC, ST-HSC, MPPs and committed progenitors were sorted and placed on adherent poly-L-lysine (PLL)-coated glass slides for $6 \mathrm{~h}$. $20 \mathrm{nM}$ TMRM was then added in the media and live cell images were acquired on a Leica SP5 confocal microscope. For MitoTracker Deep Red (Invitrogen) staining, cells were incubated at $200 \mathrm{nM}$ for $1 \mathrm{~h}$ at $37^{\circ} \mathrm{C}$.

Cell cycle staining. Sorted HSCs were fixed and permeabilized using Cytofix/Cytoperm plus kit (BD), according to the manufacturer's instruction. Fixed cells were then stained overnight with FITC Ki67 (BD) at $4{ }^{\circ} \mathrm{C}$, and $10 \mathrm{~min}$ by Hoechst 33342 (Invitrogen). 
CFSE staining. Freshly sorted LT-HSCs were incubated for $20 \mathrm{~min}$ at $37^{\circ} \mathrm{C}$ with 1:400 CFSE stock solution (Cayman chemicals; CFSE cell division assay kit). The cells were pelleted and resuspended in $1 \mathrm{ml}$ of Stemline II (Sigma) containing 10\% FBS for $20 \mathrm{~min}$ at $37^{\circ} \mathrm{C}$. Afterwards, the cells were washed twice with $1 \mathrm{ml}$ of Stemline II (Sigma) and put in culture.

HSC culture. HSCs were cultured under 'expansion' conditions in U-bottom 96-well plates for 5 days. Cultures were maintained in Stemline II (Sigma) supplemented with $10 \mu \mathrm{g} \mathrm{ml}^{-1}$ Heparin (Sigma), $100 \mathrm{ng} \mathrm{ml}^{-1}$ SCF (R\&D Systems), $2 \mathrm{ng} \mathrm{ml}^{-1}$ Flt3 ligand (R\&D), $20 \mathrm{ng} \mathrm{ml}^{-1} \mathrm{TPO}$ (R\&D Systems), $10 \mathrm{ng} \mathrm{ml}^{-1}$ FGF-1 (Invitrogen), $500 \mathrm{ng} \mathrm{ml}^{-1}$ IGFBP2 (R\&D Systems) and $100 \mathrm{ng} \mathrm{ml}^{-1}$ AngL-3 (R\&D Systems) ${ }^{31}$. At the end of the culture period, cells were stained with TMRM and analysed or sorted by flow cytometry. To induce differentiation, HSCs were cultured in a basal medium (Stemline II containing $100 \mathrm{ng} \mathrm{ml}^{-1}$ SCF and $2 \mathrm{ng} \mathrm{ml}^{-1}$ Flt3 ligand) supplemented with $20 \mathrm{ng} \mathrm{ml}^{-1} \mathrm{IL}_{-3}$ (R\&D Systems) and $100 \mathrm{ng} \mathrm{ml}^{-1}$ IL-6 (R\&D Systems). For some experiments, $5 \mu \mathrm{M}$ carbonyl cyanide 4-(trifluoromethoxy)phenylhydrazone (FCCP) (Sigma) was added at the medium. FCCP stock solution was prepared by dissolving the powder in ethanol at $10 \mathrm{mM}$ concentration.

Transplantations. C57Bl/6 Ly5.2 female mice (8-12-weeks-old) were lethally irradiated on an X-ray irradiator at 850 rads in two split doses of 425 rads separated by $3-5 \mathrm{~h}$ and transplanted with donor cells derived from C57Bl/6 Ly5.1 mice and competitor cells derived from F1 C57Bl/6 Ly5.1/5.2 mice. For LKS transplants, 1,000 LKS (TMRM ${ }^{\text {low }}$ or TMRM ${ }^{\text {high }}$ ) donor cells were transplanted together with $250 \times 10^{3}$ total BM competitor cells in recipient mice. For LKS CD150 + CD34 - ('LT-HSC') and LKS CD150 + CD34 + ('ST-HSC') transplants, 80 LT-HSC (TMRM $^{\text {low }}$ or TMRM ${ }^{\text {high }}$ ) or 80 ST-HSC $\left(\right.$ TMRM $^{\text {low }}$ or TMRM ${ }^{\text {high }}$ ) were transplanted together with $250 \times 10^{3}$ total BM competitor cells in recipient mice. Peripheral blood was collected at 4,8 and 16 weeks to determine the the percentage of chimerism. For transplantation of in vitro-cultured LT-HSCs, the progeny of 200 LT-HSCs cultured for 5 days were FACS-sorted based on their TMRM signal (TMRM low or TMRM ${ }^{\text {high }}$ ) and transplanted together with $2 \times 10^{6}$ helper cells. Helper cells were derived from BM of C57Bl/6 Ly5.1/5.2 mice that were depleted for Scal and CD150 positive cells (Miltenyi Biotech). Peripheral blood was collected at 4,8 and 16 weeks to determine the percentage of chimerism. For the CFSE-TMRM transplants, LT-HSCs were sorted and stained for CFSE. At the end of a 2-day culture period, cell progeny having undergone one division were stained with TMRM and resorted based on TMRM ${ }^{\text {low }}$ and TMRM ${ }^{\text {high }}$ signals. Each recipient mouse was injected with 100 cells of either population together with $2 \times 10^{6}$ helper cells. Peripheral blood was collected at 4,8 and 16 weeks to determine the percentage of chimerism.

For transplants of cultured HSC exposed to the uncoupler FCCP, 100 LT-HSC:TMRM ${ }^{\text {low }}$ cells were cultured for 5 days under differentiation-inducing conditions in the presence or absence of $5 \mu \mathrm{M}$ FCCP. FCCP was replenished every $24 \mathrm{~h}$. Cell progeny were transplanted in lethally irradiated recipient mice together with $2 \times 10^{6}$ helper cells. Peripheral blood was collected at $4,8,12$ and 16 weeks to determine the level of chimerism.

For secondary BM transplantation, secondary C57Bl/6 Ly5.2 recipient mice were lethally irradiated as described above and transplanted with 3 million BM cells from the primary recipients. Peripheral blood was collected at 4, 8 and 16 weeks to determine the level of chimerism.

HSC homing. C57Bl/6 Ly5.2 mice were lethally irradiated on an X-ray irradiator at 850 rads in two split doses of 425 rads separated by $3-5 \mathrm{~h}$ and transplanted with donor cells derived from C57Bl/6 Ly5.1/5.2. Four thousand LT-HSC (TMRM ${ }^{\text {low }}$ or $\mathrm{TMRM}^{\text {high }}$ ) were transplanted in recipient mice. BM was collected after $16 \mathrm{~h}$ to determine the percentage of homed donor-derived cells ${ }^{42}$.

Analysis of mitochondrial mass and autophagy level. For the live cell mitochondrial mass measurements, freshly isolated BM cells from R26-Mito-EGFP mice were stained with specific antibodies for the different haematopoietic stem/progenitor cell compartments. Labelled cells were FACS-sorted and live cells analysed by spinning disk confocal microscopy on a Visitron CSU W1 microscope. Mitochondrial mass was quantified as the intensity-thresholded area of mito-EGFP signal. For the autophagy-level study, freshly isolated BM cells were stained with specific antibodies for the different haematopoietic stem/progenitor cell compartments. LT-HSC:TMRM ${ }^{\text {low }}$ cells were FACS-sorted, separated in halves and cultured in differentiation-inducing medium, defined as basal medium (Stemline II containing $100 \mathrm{ng} \mathrm{ml}^{-1} \mathrm{SCF}$ and $2 \mathrm{ng} \mathrm{ml}^{-1}$ Flt3 ligand) supplemented with $20 \mathrm{ng} \mathrm{ml}^{-1} \mathrm{IL}-3$ (R\&D Systems) and $100 \mathrm{ng} \mathrm{ml}^{-1}$ IL-6 (R\&D Systems). Cells were cultured for 5 days either with addition of $5 \mu \mathrm{M} \mathrm{FCCP}$ or an equivalent amount of vehicle (EtOH). On the last day of culture, each population was separated in halves and further cultured for $4 \mathrm{~h}$ in the following conditions: FCCP-treated cells were either cultured with $5 \mu \mathrm{M}$ FCCP and $1 \mu \mathrm{M}$ Pepstatin A or with $5 \mu \mathrm{M}$ FCCP and an equivalent amount of vehicle (DMSO), while the control vehicle-treated cells were either with vehicle $(\mathrm{EtOH})$ and $1 \mu \mathrm{M}$ Pepstatin A or with vehicle alone (EtOH + DMSO). After $4 \mathrm{~h}$, the cells were fixed with $4 \%$ formaldehyde for $5 \mathrm{~min}$ at room temperature, permeabilized with $\mathrm{MeOH}$ for $5 \mathrm{~min}$, washed with $\mathrm{PBS} \mathrm{pH} 7.4$ and blocked for $1 \mathrm{~h}$ with $2 \%$ goat serum in PBS pH 7.4 with slight agitation. The cells were incubated for an additional $2 \mathrm{~h}$ with the rabbit anti-LC3B (Abcam, ab51520) and mouse anti-TOMM20 (Abcam ab56783) primary antibodies with slight agitation, washed three times with $\mathrm{PBS} \mathrm{pH}$ 7.4, incubated with goat anti-mouse Alexa488 and goat anti-rabbit Alexa647 secondary antibodies with slight agitation for $1 \mathrm{~h}$ and washed three times with PBS pH 7.4. Stained cells were imaged by spinning disk confocal microscopy on a Visitron CSU W1 microscope. Mitochondrial and autophagosomal mass were quantified as the intensity-thresholded area of TOMM20 and LC3B signal, respectively.

In vivo activation of HSC. HSC were activated to exit dormancy by IFN- $\alpha$ treatment following published protocols ${ }^{35}$. Briefly, subcutaneous injections in C57Bl/6J mice were carried out with 10,000 U of IFN- $\alpha$ (R\&D systems) 48 and $24 \mathrm{~h}$ prior to $\mathrm{BM}$ extraction. Control mice were injected with an equivalent volume of the vehicle (PBS $+0.1 \% \mathrm{BSA})$.

QPCR analysis. LT-HSCs were lysed after 5 days culture in lysis buffer from ZR RNA MicroPrep (Zymo Research) and RNA extraction was performed accordingly to the manufacturer's instruction. RNA was eluted and resuspended in $6 \mu \mathrm{l}$ of $\mathrm{H}_{2} \mathrm{O}$. Four microlitres of RNA was retro-transcribed to cDNA with Vilo SScript system (Invitrogen). Subsequently cDNA was diluted five times in water. For QPCR $1.5 \mu \mathrm{l}$ of cDNA, $5 \mu \mathrm{l}$ of Power Syber Green mastermix (Applied Biosystem) and $200 \mathrm{nM}$ of primers were added to a final volume of $10 \mu \mathrm{l}$ for each reaction. The reactions were performed on 7900HT system (Applied Biosystem). Primer sequences $\left(5^{\prime}-3^{\prime}\right)$ are the following: LC3-F GTCACCCAGGCGAGTTACC; LC3-R TTACAGCGGTCGGCGAAG; Sqstm1-F GCTGAAGGAAGCTGCCCTAT; Sqstm1-R TTGGTCTGTAGGAGCCTGGT; Park2-F CCGAATCACCTGACGGT TCA; Park2-R TCTGGCTGCTTCTGAATCCC; Arbp-F AGATTCGGGATATGC TGTTGG; Arbp-R AAAGCCTGGAAGAAGGAGGTC.

Statistics. Data were statistically analysed by Student's $t$-test, one-way ANOVA followed by Bonferroni's multiple comparison test and Mann-Whitney test.

Data availability. The authors declare that all relevant data are contained within the article and Supplementary files, or are available from the authors on request.

\section{References}

1. Suda, T., Takubo, K. \& Semenza, G. L. Metabolic regulation of hematopoietic stem cells in the hypoxic niche. Cell Stem Cell 9, 298-310 (2011).

2. Hofmeister, C. C., Zhang, J., Knight, K. L., Le, P. \& Stiff, P. J. Ex vivo expansion of umbilical cord blood stem cells for transplantation: growing knowledge from the hematopoietic niche. Bone Marrow Transplant 39, 11-23 (2007).

3. Wagner, J. E. et al. Transplantation of unrelated donor umbilical cord blood in 102 patients with malignant and nonmalignant diseases: influence of CD34 cell dose and HLA disparity on treatment-related mortality and survival. Blood 100, 1611-1618 (2002).

4. Ballen, K. K., Gluckman, E. \& Broxmeyer, H. E. Umbilical cord blood transplantation: the first 25 years and beyond. Blood 122, 491-498 (2013).

5. Boitano, A. E. et al. Aryl hydrocarbon receptor antagonists promote the expansion of human hematopoietic stem cells. Science 329, 1345-1348 (2010)

6. Fares, I. et al. Cord blood expansion. Pyrimidoindole derivatives are agonists of human hematopoietic stem cell self-renewal. Science 345, 1509-1512 (2014).

7. Takubo, K. et al. Regulation of glycolysis by pdk functions as a metabolic checkpoint for cell cycle quiescence in hematopoietic stem cells. Cell Stem Cell 12, 49-61 (2013)

8. Ito, K. \& Suda, T. Metabolic requirements for the maintenance of self-renewing stem cells. Nat. Rev. Mol. Cell Biol. 15, 243-256 (2014).

9. Parmar, K., Mauch, P., Vergilio, J. A., Sackstein, R. \& Down, J. D. Distribution of hematopoietic stem cells in the bone marrow according to regional hypoxia. Proc. Natl. Acad. Sci. USA 104, 5431-5436 (2007).

10. Simsek, T. et al. The distinct metabolic profile of hematopoietic stem cells reflects their location in a hypoxic niche. Cell Stem Cell 7, 380-390 (2010).

11. Yu, W. M. et al. Metabolic regulation by the mitochondrial phosphatase PTPMT1 is required for hematopoietic stem cell differentiation. Cell Stem Cell 12, 62-74 (2013).

12. Ito, K. et al. Regulation of oxidative stress by ATM is required for self-renewal of haematopoietic stem cells. Nature 431, 997-1002 (2004).

13. Ito, K. et al. Reactive oxygen species act through p38 MAPK to limit the lifespan of hematopoietic stem cells. Nat. Med. 12, 446-451 (2006).

14. Tothova, Z. et al. FoxOs are critical mediators of hematopoietic stem cell resistance to physiologic oxidative stress. Cell 128, 325-339 (2007).

15. Chen, C. et al. TSC-mTOR maintains quiescence and function of hematopoietic stem cells by repressing mitochondrial biogenesis and reactive oxygen species. J. Exp. Med. 205, 2397-2408 (2008).

16. Mantel, C. R. et al. Enhancing hematopoietic stem cell transplantation efficacy by mitigating oxygen shock. Cell 161, 1553-1565 (2015). 
17. Gan, B. et al. Lkb1 regulates quiescence and metabolic homeostasis of haematopoietic stem cells. Nature 468, 701-704 (2010).

18. Gurumurthy, S. et al. The Lkb1 metabolic sensor maintains haematopoietic stem cell survival. Nature 468, 659-663 (2010).

19. Nakada, D., Saunders, T. L. \& Morrison, S. J. Lkb1 regulates cell cycle and energy metabolism in haematopoietic stem cells. Nature 468, 653-658 (2010).

20. Mortensen, M. et al. The autophagy protein Atg7 is essential for hematopoietic stem cell maintenance. J. Exp. Med. 208, 455-467 (2011).

21. Kiel, M. J., Yilmaz, O. H., Iwashita, T., Terhorst, C. \& Morrison, S. J. SLAM family receptors distinguish hematopoietic stem and progenitor cells and reveal endothelial niches for stem cells. Cell 121, 1109-1121 (2005).

22. Wilson, A. et al. Hematopoietic stem cells reversibly switch from dormancy to self-renewal during homeostasis and repair. Cell 135, 1118-1129 (2008).

23. Hattori, F. et al. Nongenetic method for purifying stem cell-derived cardiomyocytes. Nat. Methods 7, 61-66 (2010)

24. Folmes, C. D. et al. Somatic oxidative bioenergetics transitions into pluripotency-dependent glycolysis to facilitate nuclear reprogramming. Cell Metab. 14, 264-271 (2011).

25. Mantel, C., Messina-Graham, S. \& Broxmeyer, H. E. Upregulation of nascent mitochondrial biogenesis in mouse hematopoietic stem cells parallels upregulation of CD34 and loss of pluripotency: a potential strategy for reducing oxidative risk in stem cells. Cell Cycle 9, 2008-2017 (2010).

26. Abe, T. et al. Establishment of conditional reporter mouse lines at ROSA26 locus for live cell imaging. Genesis 49, 579-590 (2011).

27. Miyamoto, K. et al. Foxo3a is essential for maintenance of the hematopoietic stem cell pool. Cell Stem Cell 1, 101-112 (2007).

28. Jensen, K. S. et al. FoxO3A promotes metabolic adaptation to hypoxia by antagonizing Myc function. EMBO J. 30, 4554-4570 (2011).

29. Yamamoto, R. et al. Clonal analysis unveils self-renewing lineage-restricted progenitors generated directly from hematopoietic stem cells. Cell 154, $1112-1126$ (2013).

30. Gekas, C. \& Graf, T. CD41 expression marks myeloid-biased adult hematopoietic stem cells and increases with age. Blood 121, 4463-4472 (2013).

31. Zhang, C. C. et al. Angiopoietin-like proteins stimulate ex vivo expansion of hematopoietic stem cells. Nat. Med. 12, 240-245 (2006).

32. Yamazaki, S. et al. Cytokine signals modulated via lipid rafts mimic niche signals and induce hibernation in hematopoietic stem cells. EMBO J. 25, 3515-3523 (2006).

33. Lutolf, M. P., Doyonnas, R., Havenstrite, K., Koleckar, K. \& Blau, H. M. Perturbation of single hematopoietic stem cell fates in artificial niches. Integr. Biol. (Camb) 1, 59-69 (2009).

34. Takizawa, H., Regoes, R. R., Boddupalli, C. S., Bonhoeffer, S. \& Manz, M. G. Dynamic variation in cycling of hematopoietic stem cells in steady state and inflammation. J. Exp. Med. 208, 273-284 (2011).

35. Essers, M. A. et al. IFNalpha activates dormant haematopoietic stem cells in vivo. Nature 458, 904-908 (2009).

36. Poot, M. et al. Analysis of mitochondrial morphology and function with novel fixable fluorescent stains. J. Histochem. Cytochem. 44, 1363-1372 (1996).

37. Kondoh, H. et al. A high glycolytic flux supports the proliferative potential of murine embryonic stem cells. Antioxid. Redox Signal 9, 293-299 (2007).

38. Strappazzon, F. et al. AMBRA1 is able to induce mitophagy via LC3 binding, regardless of PARKIN and p62/SQSTM1. Cell Death Differ. 22, 419-432 (2015).

39. Warr, M. R. et al. FOXO3A directs a protective autophagy program in haematopoietic stem cells. Nature 494, 323-327 (2013).
40. Youle, R. J. \& Narendra, D. P. Mechanisms of mitophagy. Nat. Rev. Mol. Cell Biol. 12, 9-14 (2011).

41. Rogov, V., Dotsch, V., Johansen, T. \& Kirkin, V. Interactions between autophagy receptors and ubiquitin-like proteins form the molecular basis for selective autophagy. Mol. Cell 53, 167-178 (2014).

42. Carbonneau, C. L. et al. Ionizing radiation-induced expression of INK4a/ARF in murine bone marrow-derived stromal cell populations interferes with bone marrow homeostasis. Blood 119, 717-726 (2012).

\section{Acknowledgements}

We thank Marlen Knobloch, Adrian Ranga and Michael Snyder for valuable feedback on the manuscript. We also thank Miguel Garcia, Lopes Telma, Tuzin Loic and Gonzalo Tapia from the École Polytechnique Fédérale de Lausanne (EPFL) flow cytometry core facility; Yazdah Panah Benyamin from the Laboratory of Virology and Genetics at EPFL for help in the RNA purification; Emilie Gesina, Ferrand Giselle, Bouaziz Mohamed and Bah Ibrhaima Aliou from the Center of Phenogenomics at EPFL for support with the animal work. This work was funded by a Swiss National Science Foundation Sinergia grant (CRSII3 147684), a grant from the 3R Research Foundation and a Swiss National Science Foundation grant (CR33I3_153316) to M.P.L., as well as a grant from the Fondation Machaon to O.N. O.N. and V.C. partially funded by the José Carreras Leukemia Foundation/European Hematology Association Fellowship. J.A. is the Nestlé Chair in Energy Metabolism and his work is supported by grants from the EPFL, and the Swiss National Science Foundation (31003A-140780).

\section{Author contributions}

N.V., M.G., M.P.L., O.N. and G.N. conceived ideas, designed experiments, analysed results and wrote the manuscript. N.V., M.G., G.N. and O.N. performed the experiments. V.C. and A.R. contributed substantially to in vivo experiments. S.G. performed gene expression analyses. J.A. conceived ideas, provided key reagents and comprehensively contributed to experimental design. All authors edited and reviewed the final manuscript.

\section{Additional information}

Supplementary Information accompanies this paper at http://www.nature.com/ naturecommunications

Competing financial interests: N.V., M.P.L., J.A., O.N., M.G. are authors on a patent entitled 'Methods \& compounds useful in hematopoietic stem cell medicine' patent number P1828EP00 (2016). The remaining authors declare no competing financial interests.

Reprints and permission information is available online at http://npg.nature.com/ reprintsandpermissions/

How to cite this article: Vannini, N. et al. Specification of haematopoietic stem cell fate via modulation of mitochondrial activity. Nat. Commun. 7, 13125 doi: $10.1038 /$ ncomms13125 (2016).

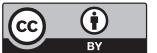

This work is licensed under a Creative Commons Attribution 4.0 International License. The images or other third party material in this article are included in the article's Creative Commons license, unless indicated otherwise in the credit line; if the material is not included under the Creative Commons license, users will need to obtain permission from the license holder to reproduce the material. To view a copy of this license, visit http://creativecommons.org/licenses/by/4.0/

(C) The Author(s) 2016 